\title{
List of Reviewers for this Special Issue
}

Journal of International Business Studies (2014) 45, I06 I. doi: I 0. I057/jibs.20 I4.44

The guest editors wish to acknowledge and thank members of the Editorial Review Board and the scholars listed below who have contributed their time to review manuscripts for this "Governments as Owners: Globalizing State-Owned Enterprises" Special Issue.

Aguilera, Ruth
Aharoni, Yair
Alon, Ilan
Belderbos, René
Benito, Gabriel
Bertrand, Olivier
Bucheli, Marcelo
Chan, Christine
Chi, Tailan
Choudhury, Prithwiraj
Cossin, Didier
Cui, Lin
Dastidar, Protiti
Deng, Ping
Doh, Jonathan
Domadenik, Polona
Dow, Douglas
Driffield, Nigel
Duanmu, Jing-Lin
Eapen, Alex
Eden, Lorraine
Fauver, Larry
Fleury, Maria Teresa
Garretsen, Harry
Gaur, Ajai
Gimeno, Javier

Gubbi, Sathyajit

Guedhami, Omrane

Hitt, Michael

Hoskisson, Robert

Jiang, Fuming

Jiang, Guo-Liang

Kafouros, Mario

Kang, Jun-Koo

Kim, Heechun

Kumar, Praveen

Kumar, Vikas

Kwok, Chuck

Lazzarini, Sergio

Li, Weiwen

Lu, Jiantao

Luo, Yadong

McDermott, Gerald

Merchant, Hemant

Mesquita, Luiz

Meyer, Klaus

Moffett, Michael

Monaldi, Francisco

Mudambi, Ram

Nachum, Lilac

Narula, Rajneesh

Nelson, Roy

Nguyen, Bang Dang
Peng, Mike

Petitt, Barbara

Puffer, Sheila

Rademaker, Linda

Ramamurti, Ravi

Roth, Kendall

Sambharya, Rakesh

Schotter, Andreas

Sleuwaegen, Leo

Spicer, Andrew

Sun, Pei

Sun, Sunny

Thun, Eric

Van Tulder, Rob

Voss, Hinrich

Wan, William

Wan, Wongsunwai

Wang, Chengqi

Washburn, Nathan

Wells, Louis

$\mathrm{Wu}$, Hamilton

$\mathrm{Wu}$, Jie

Zhang, Kevin

Zhao, Hongxin

Zhao, Minyuan

Zhao, Zheng

Zheng, Qinqin 